\title{
Paternal age, size at birth, and size in young adulthood - risk factors for schizophrenia
}

Finn Rasmussen

Child and Adolescent Public Health Epidemiology Group, Department of Public Health Sciences, Karolinska Institute, Norrbacka, SE-17176 Stockholm, Sweden

(Correspondence should be addressed to F Rasmussen; Email: finn.rasmussen@ki.se)

\begin{abstract}
It is appropriate to consider schizophrenia a neurodevelopmental disorder with its pathogenesis going back to gestation and early childhood. Schizophrenia is a rare disease and large cohorts are needed to study its etiology. The aim of this paper is to review the results of recent Swedish record-linkage studies with a focus on: (i) measures of fetal and childhood growth in relation to schizophrenia in adulthood and (ii) paternal age in relation to schizophrenia. A record-linkage was created between national registers, including the Medical Birth Register, the Military Service Conscription Register, and the Inpatient Hospital Discharge Register. More than 700000 subjects born between 1973 and 1980 were followed in these registers from birth to 31 December 2001/2002. The results showed no evidence of an association between birth weight and schizophrenia. An association of birth length with schizophrenia was observed, with short babies showing the highest risk. Short stature and low BMI in young adulthood were associated with increased risk. Short babies who became tall, or developed high BMI as adults, were not at increased risk. In fully adjusted analyses, the risk of schizophrenia was 4.62 (95\% confidence interval : 2.28;9.36) times higher in subjects whose fathers were $\geq 50$ years old and at time of conception than in subjects whose fathers were 21-24 years old. Growth and development in fetal life and childhood are influencing the risk of schizophrenia in adulthood, but the underlying causal pathways are still unknown. De novo mutations in the germ cells of older fathers may play a causal role in the etiology of some cases of schizophrenia.
\end{abstract}

European Journal of Endocrinology 155 S65-S69

\section{Introduction}

Schizophrenia is an etiologically heterogeneous disease, which usually becomes manifest in adolescence or early adulthood with onset of psychotic symptoms. Common psychotic symptoms are auditory hallucinations and delusions; other more unspecific symptoms include social withdrawal, flattened affect, poor motivation, and depressed mood. It is appropriate to consider schizophrenia a neurodevelopmental disorder with its pathogenesis going back to gestation and early childhood (1). The etiology of schizophrenia is not well understood, although genetic, as well as environmental, factors are important (2). The inheritance seems to be polygenetic, but several studies have recently indicated that de novo mutations in paternal germ cells may also be important, especially in cases of schizophrenia lacking a family history (3).

This paper was presented at the 4th Ferring Pharmaceuticals International Paediatric Endocrinology Symposium, Paris (2006). Ferring Pharmaceuticals has supported the publication of these proceedings.
Adverse events during pregnancy and delivery (infections, nutritional deficiency, maternal bleeding, pre-eclampsia, gestational diabetes, rhesus incompatibility, and psychosocial stress) play a role, perhaps by increasing susceptibility to genetic or environmental factor later during the life course (4). Impaired fetal growth, as indexed by low birth weight and birth length for gestational age, comprises putative risk factors for schizophrenia (5), but as reported later the evidence is far from conclusive. Infections during pregnancy, such as influenza (6), rubella, toxoplasmosis, and herpes simplex type-2 are putative risk factors for schizophrenia along with severe maternal undernutrition during pregnancy (1). Studies on women who conceived their offspring or were in early pregnancy at the time of the Dutch famine in the winter of 1944-1945 indicated that severe maternal undernutrition increases the risk of schizophrenia in the offspring (7). Similar results were reported in a study examining the effects of the Chinese famine in 1959-1961 (8). However, severe psychosocial stress during pregnancy is a recognized risk factor, which must also be present under such extreme conditions as famine. Interestingly, a small study, including schizophrenic patients and healthy control (comparison) subjects 
either exposed or not exposed during the first trimester of fetal life to the Dutch Hunger Winter were examined in adulthood by magnetic resonance imaging. The results indicated that factors affecting brain growth during the first trimester of gestation may be risk factors for the development of schizophrenia later in life (9).

Delayed motor and speech development in early childhood, urban place of living (10), and cannabis abuse in late childhood are also the risk factors for schizophrenia (1). Poor school performance and poor results in intelligence tests may also be the risk factors or perhaps more likely prodromal symptoms of schizophrenia becoming manifest later in life (11). As described in greater details later, low body mass index (BMI) $(12,13)$ and short stature in adolescence (13) have recently been recognized as risk factors for schizophrenia.

This short paper focuses on: (i) measures of fetal and childhood growth in relation to schizophrenia in adulthood and (ii) paternal age in relation to schizophrenia. It will summarize results from recent research conducted on large birth cohorts from Sweden $(3,10$, $11,13-15)$ and briefly relate the findings to most recent knowledge from the literature.

\section{Materials and methods}

\section{Study populations}

The associations of fetal and childhood growth and body size in young adulthood with the risk of schizophrenia were studied in a Swedish cohort (study) including 719476 singleton males and females, born in Sweden between 1973 and 1980 (13). These study subjects were alive and resident in Sweden at the age of 16 years. Subjects who had been admitted to hospital for any type of psychosis prior to the age of 16 years were excluded.

Associations of paternal age with risk of schizophrenia were also studied in subjects born in Sweden during 1973-1980. Subjects who had died, emigrated, or had received inpatient hospital care for any type of psychosis before the age of 16 years were excluded. Information about paternal age, maternal age, and parity was obtained from the Multi-Generation Register (see below) and 754330 subjects had complete data on all variables used (3).

\section{Information sources}

The Swedish studies summarized and reviewed are based on record-linkages of several national registers: the Medical Birth Register, Military Service Conscription Register, the Hospital Inpatient Discharge Register, the Multi-Generation Register, and the Population and Housing Censuses.

The Medical Birth Register is a national register including more than $99 \%$ of all births in Sweden since 1973 (16). This information source includes data on place and season of birth, birth weight, birth length, gestational age, head circumference, Apgar score, International Classification of Diseases (ICD)-diagnoses of maternal diseases and complications during pregnancy or delivery (pre-eclampsia and maternal hypertension, ante-partum hemorrhage, operative (Cesarean) delivery, prolonged labor, and maternal diabetes), and ICDdiagnoses of diseases in the newborn baby.

In the past (and during the study period), all men in Sweden were required by law to undergo a military service conscription examination at the age of 18 years unless they suffered from a severe chronic medical condition or handicap that had to be verified by a medical certificate. About $90 \%$ of all Swedish men are included in the Military Service Conscription Register. Height and weight are measured at conscription at the age of 18 years and BMI is calculated, and according to it subjects are defined as underweight $\left(<18.5 \mathrm{~kg} / \mathrm{m}^{2}\right)$, normal weight $\left(18.5-24.9 \mathrm{~kg} / \mathrm{m}^{2}\right)$, overweight $(25.0-$ $\left.30.0 \mathrm{~kg} / \mathrm{m}^{2}\right)$, or obese $\left(>30.0 \mathrm{~kg} / \mathrm{m}^{2}\right)$.

Diagnoses of schizophrenia among inpatient cohort members were retrieved from the Hospital Inpatient Discharge Register. Hospital admissions for schizophrenia were coded using the ICD versions 9 and 10 . ICD-9 codes 295, 297-299 and ICD-10 codes F20-F29. Included in analyses are cases of schizophrenia diagnosed after the age of 16 years. Analyses using anthropometric data from conscription include cases of schizophrenia (males only) diagnosed at or after (but not before) conscription examination.

Information on age of biological parents was extracted from the Multi-Generation Register and data on parental income, highest socioeconomic index of either parent, and highest educational level of either parent was obtained from the Population and Housing Censuses as described elsewhere $(3,13)$.

\section{Statistical analyses}

Cox proportional hazards model was used to study the associations between anthropometric measures, paternal age, and risk of schizophrenia. The analyses were adjusted for year of birth, gender, socioeconomic position of parents, and other potential confounders as described later. Start of follow-up was time of birth in analyses of association of size at birth with risk of schizophrenia in young adulthood. In analyses of adult anthropometry only, start of follow-up was date of conscription. In all analyses, study subjects were followed-up to date of diagnosis of schizophrenia, date of emigration, date of death or to end of follow-up as of 31 December 2001 (3) or 2002 (13), whichever came first. Individuals with a diagnosis of schizophrenia, nonaffective/non-schizophrenic psychosis, and bipolar or severe depressive disorders diagnosed before the age of 16 years were excluded. 


\section{Results}

\section{Size at birth and schizophrenia}

Analyses based on 736 males and females with schizophrenia within the cohort including 719476 study subjects showed no consistent evidence of associations of birth weight with schizophrenia (13). However, short babies were at an increased risk of schizophrenia and gestational age/prematurity did not confound associations of birth length with schizophrenia. Hazard ratio per $10 \mathrm{~cm}$ increase in birth length was $0.53(95 \%$ confidence interval (CI): $0.31,0.91$ ). The hazard ratio for babies with a birth length of $53 \mathrm{~cm}$ or more was 0.66 (95\% CI: 0.45, 0.96) compared with those with a birth length $<49 \mathrm{~cm}$ corresponding to a $34 \%$ lower risk of schizophrenia (13). These analyses were adjusted for sex, year of birth, gestational age, birth weight, operative delivery, parity, paternal age, Apgar score, place of birth, season of birth, family history of psychosis, and childhood socioeconomic position (13).

\section{Adult body size and schizophrenia}

In analyses based on 300 males with schizophrenia from the cohort born between 1973 and 1980, height and BMI at the age of 18 years were inversely associated with risk of schizophrenia (hazard ratio for $10 \mathrm{~cm}$ increase in height: 0.81 (95\% CI: 0.67, 0.97); hazard ratio for 5 unit increase in BMI: 0.78 (95\% CI: $0.63,0.97)$ ). Small babies who went on to become tall or high BMI adults were not at increased risk of schizophrenia (13). These analyses were adjusted for calendar year, age at conscription, family history of psychosis, father's age, municipality type and size, and level of education of both parents. Further adjustments for birth measures had very little impact on these associations. The inverse associations with height appeared to be most marked in short babies, the greatest risk being in short babies who remained small in adulthood (13).

It is a possibility that some of the subjects developing schizophrenia after conscription had a sub-clinical or prodromal disease already at conscription, which may have changed their eating habits, physical activity patterns, and BMI. However, examination of associations with height and BMI were also repeated in subjects first admitted with schizophrenia after the initial 5-year period following conscription. In this restricted analyses, taller height and higher BMI at conscription were still associated with decreased risk of schizophrenia with effect sizes almost identical to those of the whole sample group (13).

\section{Paternal age and schizophrenia}

Proportional hazards regression analyses, with adjustment for a range of confounding factors described later, showed strong association between risk of schizophrenia and advancing paternal age (3). The hazards ratios were $2.32(95 \%$ CI: $1.56,3.44)$ for males and females whose fathers were 35-39 years old at time of conception, 2.08 (95\% CI: 1.25, 3.46) for those whose fathers were $40-44$ years old, 1.30 (95\% CI: $0.56,3.06)$ for those whose fathers were 45-49 years old, and 4.62 (95\% CI: $2.28 ; 9.36$ ) for those whose fathers were $\geq 50$ years old at time of conception. The reference group comprised study subjects whose fathers were 21-24 years old at their conception. Factors taken into account in the analyses were sex, age, maternal age and parity, birth weight, birth length, gestational age, urban vs rural status of place of birth, season of birth, Apgar score at 1 and 5 min after birth, multiple birth, parental death in childhood, family history of schizophrenia, and highest annual income of either parent, highest socioeconomic index of either parent, and highest educational level of either parent.

\section{Discussion}

\section{Methodological issues}

This study has some limitations. First, the Inpatient Hospital Discharge Register is an administrative register and some underreporting of cases of schizophrenia is known to exist primarily because outpatient care is not covered (17). However, in longitudinal studies, most cases of schizophrenia will appear in inpatient care. Due to the independent nature of any missing information in the Inpatient Hospital Discharge Register (diagnosis of schizophrenia) and the Military Service Conscription Register (height and weight), the net effect will be some degree of underestimation of the true associations. Secondly, data on BMI were only available from one time point (age of 18 years) and its patterns of longitudinal change are thus unknown. Thirdly, it is a limitation that schizophrenia could only be studied in young adults and that adult height and weight were only available for males. Therefore, no inferences could be made regarding possible consequences of short adult height and low adult BMI in women. Fourthly, BMI is an imperfect measure of body fatness, but nonetheless strongly correlated with other measures of body fatness (waist circumference and fat mass measured by dual energy X-ray absorptiometry, etc.).

However, this study also has several strengths. First, its very large size and population-based nature gave high statistical power to detect true associations. Secondly, the large number of cases of schizophrenia made it feasible to repeat the analyses after excluding subjects admitted to hospital because of schizophrenia within the initial 5-year period following conscription. It would therefore be too hasty to suspect that the inverse association between BMI and risk of schizophrenia appeared as an artifact due to early manifestations of 
schizophrenia, such as depressive mood and diminished appetite leading to weight loss. After these exclusions, the risk estimates (hazard ratios) were almost identical to estimates obtained from the full sample (13). The BMI-schizophrenia association, therefore, cannot be explained by reverse causality.

\section{Pre- and perinatal adversities, size at birth, growth in childhood, and schizophrenia}

The meta-analysis of prospective population-based studies, briefly referred to above, revealed that three groups of complications were significantly associated with schizophrenia (5): (i) complications of pregnancy (bleeding, diabetes, rhesus incompatibility, and preeclampsia); (ii) abnormal fetal growth and development (low birth weight, congenital malformations, and reduced head circumference); and (iii) complications of delivery (uterine atony, asphyxia, and emergency Cesarean section). Pooled estimates of effect sizes were, however, generally small and less than two. Pre- and perinatal adversities and complications may occur in up to $25 \%$ of all pregnancies (5) depending on definition, and most children do not develop schizophrenia. Further, many individuals with schizophrenia have not been subjected to recognized obstetric complications. It is obvious that obstetric complications are neither necessary nor sufficient causal factors for schizophrenia (4). It is noteworthy that the inverse association of birth weight with risk of schizophrenia reported by Cannon et al. (5) in 2002 is inconsistent with the findings of the large Swedish study reported in 2005, which showed no association of birth weight with schizophrenia (13).

Levels of insulin-like growth factor-I (IGF-I) in cord blood have been found to be associated with size at birth (18), and IGF-I serum levels have been reported to be associated with growth of legs and trunk from 5 to 10 years of age (19). Evidence from animal research, suggests that growth hormone and IGF-I are important in neurodevelopment (20), and may therefore potentially be physiological mediators of the association of short birth length with schizophrenia (21).

\section{Genetic factors, body size, and schizophrenia}

Height and BMI are to a large extent determined by genetic factors $(22,23)$ and twin studies and other family studies have consistently shown that genetic factors are important in the development of schizophrenia (24). Genes coding for proteins, such as glutamic acid decarboxylase, IGF-I, neuroregulin 1, dysbindin, COMT, the dopamine D2 receptor, and the serotonin 5-HT2A receptor may potentially play a role in the pathogenesis of schizophrenia (2). Genes coding for IGF-I are especially interesting because IGF-I seems to have a role in shaping synaptic connections or myelinization $(2,21,25)$. However, the cellular mechanisms and clinical relevance of these and other specific genes remain to be confirmed. It is possible that genetic predisposition to schizophrenia influences preand postnatal patterns of growth, which may explain the associations of body size with schizophrenia. It is an open question whether the association of birth length with schizophrenia reflects the effect of schizophrenia susceptibility genes that are closely linked with those influencing growth.

\section{Paternal age and schizophrenia}

Increased paternal age is associated with several diseases possibly due to age-associated increase in sporadic de novo mutations in male germ cells (3). The Swedish study showed a strong positive association of paternal age at conception with risk of schizophrenia in young adulthood (3) and confirms previous smaller studies of schizophrenia $(26,27)$. The paternal ageschizophrenia association is stronger in those with no family history of schizophrenia, supporting the hypothesis that accumulating de novo mutations in the germ cells of older fathers could play an important role in the etiology of schizophrenia (3).

\section{Concluding remark}

Short length at birth, short height and low BMI in young adult males, and high paternal age at conception are risk factors for schizophrenia according to Swedish studies $(3,13)$. While de novo mutations in paternal germ cells provide a reasonable or at least possible explanation for the paternal age-schizophrenia association, causal pathways explaining associations of size at birth and size in young adulthood with schizophrenia are largely unknown. However, since IGF-I is a major regulator of childhood growth (19) and is also influencing brain development in animal research (20), an intriguing hypothesis for future research is that IGF-I serves as a biological link between childhood growth, body size in adulthood and schizophrenia.

\section{References}

1 Mäki P, Veijola J, Jones PB, Murray GK, Koponen H, Tienari P, Miettunen J, Tanskanen P, Wahlberg KE, Koskinen J, Lauronen E \& Isohanni M. Predictors of schizophrenia - a review. British Medical Bulletin 2005 73-74 1-15.

2 Rapoport JL, Addington AM, Frangou S \& Psych MR. The neurodevelopmental model of schizophrenia: update 2005. Molecular Psychiatry 200510 434-449.

3 Sipos A, Rasmussen F, Harrison G, Tynelius P, Lewis G, Leon DA \& Gunnell D. Paternal age and schizophrenia: a population based cohort study. British Medical Journal 20043291070.

4 Clarke MC, Harley M \& Cannon M. The role of obstetric events in schizophrenia. Schizophrenia Bulletin 200632 3-8.

5 Cannon M, Jones PB \& Murray RM. Obstetric complications and schizophrenia: historical and meta-analytic review. American Journal of Psychiatry 2002159 1080-1092. 
6 Brown AS, Begg MD, Gravenstein S, Schaefer CA, Wyatt RJ, Bresnahan Babulas VP \& Susser ES. Serologic evidence of prenatal influenza in the etiology of schizophrenia. Archives of General Psychiatry 200461 774-780.

7 Susser E, Neugebauer R, Hoek HW, Brown AS, Lin S, Labovitz D \& Gorman JM. Schizophrenia after prenatal famine. Further evidence. Archives of General Psychiatry 199653 25-31.

8 St Clair D, Xu M, Wang P, Yu Y, Fang Y, Zhang F, Zheng X, Gu N, Feng G, Sham P \& He L. Rates of adult schizophrenia following prenatal exposure to the Chinese famine of 1959-1961. Journal of the American Medical Association 2005294 557-562.

9 Hulshoff Pol HE, Hoek HW, Susser E, Brown AS, Dingemans A, Schnack HG, van Haren NE, Pereira Ramos LM, Gispen-de Wied CC \& Kahn RS. Prenatal exposure to famine and brain morphology in schizophrenia. American Journal of Psychiatry 2000 157 1170-1172.

10 Harrison G, Fouskakis D, Rasmussen F, Tynelius P, Sipos A \& Gunnell D. Association between psychotic disorder and urban place of birth is not mediated by obstetric complications or childhood socio-economic position: a cohort study. Psychological Medicine 200333 723-731.

11 Gunnell D, Harrison G, Rasmussen F, Fouskakis D \& Tynelius P. Associations between premorbid intellectual performance, earlylife exposures and early-onset schizophrenia. Cohort study. British Journal of Psychiatry $2002 \mathbf{1 8 1} 298-305$.

12 Sørensen HJ, Mortensen EL, Reinisch JM \& Mednick SA. Height, weight and body mass index in early adulthood and risk of schizophrenia. Acta Psychiatrica Scandinavica 2006114 49-54.

13 Gunnell D, Harrison G, Whitley E, Lewis G, Tynelius P \& Rasmussen F. The association of fetal and childhood growth with risk of schizophrenia. Cohort study of 720000 Swedish men and women. Schizophrenia Research 200579 315-322.

14 Fouskakis D, Gunnell D, Rasmussen F, Tynelius P, Sipos A \& Harrison $\mathrm{G}$. Is the season of birth association with psychosis due to seasonal variations in foetal growth or other related exposures? A cohort study. Acta Psychiatrica Scandinavica 2004109 259-263.

15 Gunnell D, Rasmussen F, Fouskakis D, Tynelius P \& Harriso G. Patterns of fetal and childhood growth and the development of psychosis in young males: a cohort study. American Journal of Epidemiology $2003 \mathbf{1 5 8}$ 291-300.

16 Cnattingius S, Ericson A, Gunnarskog J \& Källen B. A quality study of a medical birth registry. Scandinavian Journal of Social Medicine $199018143-148$.

17 Dalman C, Broms J, Cullberg J \& Allebeck P. Young cases of schizophrenia identified in a national inpatient register - are the diagnoses valid? Social Psychiatry and Psychiatric Epidemiology $200237527-531$.
18 Ong K, Kratzsch J, Kiess W, Costello M, Scott C \& Dunger D. Size at birth and cord blood levels of insulin, insulin-like growth factor I (IGF-I), IGF-II, IGF-binding protein-1 (IGFBP-1), IGFBP-3, and the soluble IGF-II/mannose-6-phosphate receptor in term human infants. The ALSPAC Study Team. Avon Longitudinal Study of Pregnancy and Childhood. Journal of Clinical Endocrinology and Metabolism 200085 4266-4269.

19 Rogers I, Metcalfe C, Gunnell D, Emmett P, Dunger D \& Holly J. IGF-I and growth in height, leg length and trunk length between ages 5 and 10 years. Journal of Clinical Endocrinology 200691 2514-2619.

20 Niblock MM, Brunso-Bechtold JK \& Riddle DR. Insulin-like growth factor I stimulates dendritic growth in primary somatosensory cortex. Journal of Neuroscience 200020 4165-4176.

21 Gunnell D \& Holly JMP. Do insulin-like-growth factors underlie associations of birth complications, fetal and pre-adult growth with schizophrenia? Schizophrenia Research 200467 309-311.

22 Silventoinen K, Krueger RF, Bouchard TJ Jr, Kaprio J \& McGue M. Heritability of body height and educational attainment in an international context: comparison of adult twins in Minnesota and Finland. American Journal of Human Biology 200416 544-555.

23 Stunkard AJ, Harris JR, Pedersen NL \& McClearn GE. The bodymass index of twins who have been reared apart. New England Journal of Medicine 1990322 1483-1487.

24 Riley BP \& Kendler KS. Schizophrenia: genetics. In Kaplan and Sadock's Comprehensive Textbook of Psychiatry, pp 1354-1370. Eds BJ Sadock \& VA Sadock. Philadelphia, PA: Lippincott-Williams \& Wilkins, 2006.

25 Bondy CA. Transient IGF-I gene expression during the maturation of functionally related central projection neurons. Journal of Neuroscience 199111 3442-3455.

26 Dalman C \& Allebeck P. Paternal age and schizophrenia: further support for an association. American Journal of Psychiatry 2002 159 1591-1592.

27 Brown AS, Schaefer CA, Wyatt RJ, Begg MD, Goetz R, Bresnahan MA, Harkavy-Friedman J, Gorman JM, Malaspina D \& Susser ES. Paternal age and risk of schizophrenia in adult offspring. American Journal of Psychiatry 2002159 1528-1533.

Received 26 June 2006

Accepted 20 July 2006 